Supporting Information for

\title{
Temperature and Nuclear Quantum Effects on the Stretching Modes of the Water Hexamer
}

\author{
Nagaprasad Reddy Samala, Noam Agmon \\ The Fritz Haber Research Center, Institute of Chemistry, The Hebrew University of Jerusalem \\ 91904, Israel.
}

\section{Contents:}

\begin{tabular}{lll} 
Item & Description & Pages \\
\hline Table S1 & $\begin{array}{l}\text { Comparison of experimental book isomer frequencies with theoretical } \\
\text { predictions from various MB-PES }\end{array}$ & $\mathrm{S} 2$ \\
\hline Table S2 & $\begin{array}{l}\text { Comparison of the above with frequencies calculated from various quantum } \\
\text { chemistry theories }\end{array}$ & $\mathrm{S} 3$ \\
\hline Table S3 & $\begin{array}{l}\text { Comparison with experiment of the MB-pol frequencies when shifted to best-fit } \\
\text { the various quantum chemistry theories of Table S2 }\end{array}$ & $\mathrm{S} 4$ \\
\hline Figure S1 & All H-atom and partial VACF vibrational spectra of the cage isomer at 10K & $\mathrm{S} 5$ \\
\hline Figure S2 & Same at 30K & $\mathrm{S} 6$ \\
\hline Figure S3 & Same at 50K & $\mathrm{S} 7$ \\
\hline Figure S4 & Same at 70K & $\mathrm{S} 8$ \\
\hline Figure S5 & All H-atom and partial VACF vibrational spectra of ring isomer at 10K & $\mathrm{S} 9$ \\
\hline Figure S6 & All H-atom and partial VACF vibrational spectra of prism isomer at 10K & $\mathrm{S} 10$ \\
\hline
\end{tabular}


Table S1. Comparison with experiment of the book-isomer OH-stretch spectrum calculated on various MB-PESs (no scaling factors apply here). The correlation between theoretically calculated and experimentally determined frequencies used for the error estimates in $\mathrm{HBed}-\mathrm{OH}$ frequencies is explained in the footnotes.

\begin{tabular}{|c|c|c|c|c|c|c|}
\hline mode & Exper. $^{\mathrm{a}}$ & $\mathrm{WHBB}^{\mathrm{b}}$ & E3B2 ${ }^{c}$ & $\begin{array}{l}\text { shifted } \\
\text { E3B2 }^{\mathrm{d}}\end{array}$ & MB-pol/LM ${ }^{\mathrm{e}}$ & $\begin{array}{l}\text { shifted MB- } \\
\text { pol/VACF }^{\mathrm{f}}\end{array}$ \\
\hline $1-4^{g}$ & 3711 & 3712 & 3746 & & 3720 & 3707 \\
\hline 5 & & & 3716 & & 3713 & 3693 \\
\hline 6 & 3572 & 3551 & 3560 & 3620 & 3572 & 3584 \\
\hline 7 & 3464 & 3436 & 3421 & 3481 & 3434.5 & 3467 \\
\hline 8 & & & 3380 & 3440 & 3431.7 & 3452 \\
\hline 9 & 3423 & 3397 & 3309 & 3369 & 3416.8 & 3410 \\
\hline 10 & 3327 & 3336 & 3276 & 3336 & 3285.1 & 3327 \\
\hline 11 & 3287 & 3313 & 3170 & 3230 & 3268 & 3278 \\
\hline 12 & 3201 & 3276 & 3105 & 3165 & 3246.4 & 3218 \\
\hline bend $* * 2$ & 3169 & 3170 & & & 3147 & \\
\hline meanD $^{\mathrm{h}}$ & 0 & 5 & -60 & 35 & -6.1 & 0.5 \\
\hline MAD $^{\text {h }}$ & 0 & 27 & 70 & 35 & 22 & 9.2 \\
\hline $\max D^{h}$ & 0 & 75 & 117 & 57 & 45 & 17 \\
\hline
\end{tabular}

${ }^{a}$ Gas-phase, Table 1 of Diken et al. ${ }^{13}$ Band 5 merges with 1-4, bands 7-8 merge into a single band. ${ }^{\mathrm{b}}$ Digitized from Figure 1 of Wang and Bowman. ${ }^{27}$ Bands 5 and 8 not seen here (see above). ${ }^{\mathrm{c}}$ From Figure 7 of Kananenka et al. ${ }^{24}$ No bend overtone (bend $* * 2$ ) seen in classical MD. ${ }^{\mathrm{d}} \mathrm{E} 3 \mathrm{~B} 2 \mathrm{HBed} \mathrm{OH}$ frequencies shifted by - meanD $=60 \mathrm{~cm}^{-1}$, to best fit experiment.

${ }^{\mathrm{e}} \mathrm{MB}$-pol with the Local Monomer (LM) approximation. From Table S4 of Brown et al. ${ }^{29}$ Bend overtones and combinations were not assigned there, so we cite here the average of the two strongest bands below $3200 \mathrm{~cm}^{-1}$ as the bend overtone. A weak band at $3251.8 \mathrm{~cm}^{-1}$ is not assigned. ${ }^{\mathrm{f}}$ Our results, shifted by $208 \mathrm{~cm}^{-1}$. No bend overtone seen in classical MD.

${ }^{\mathrm{g}}$ Bands 1-4 are averaged together.

${ }^{\mathrm{h}}$ Errors with respect to experiment for modes 6,7, 9-12 (i.e., all available HBed-OH stretching modes). Whenever modes 7 and 8 are resolved, they are averaged together for this comparison. 
Table S2. Comparison with experiment of the book-isomer $\mathrm{OH}$-stretch spectrum calculated by scaled-harmonic quantum chemistry methods.

\begin{tabular}{|l|l|l|l|l|}
\hline mode & Exper. $^{\mathrm{a}}$ & $\begin{array}{l}\mathrm{MP}^{\mathrm{b}} \\
6-311+\mathrm{G}^{* *}\end{array}$ & $\begin{array}{l}\mathrm{MP}^{\mathrm{c}} \\
\text { aug-cc-pVDZ }\end{array}$ & $\mathrm{VPT}^{\mathrm{d}}$ \\
\hline $1-4^{\mathrm{e}}$ & 3711 & 3711 & 3763 & 3693 \\
\hline 5 & -- & 3696 & 3755 & 3686 \\
\hline 6 & 3572 & 3594 & 3617 & 3546 \\
\hline 7 & 3464 & 3472 & 3473 & 3415 \\
\hline 8 & & 3450 & 3458 & 3398 \\
\hline 9 & 3423 & 3406 & 3405 & 3356 \\
\hline 10 & 3327 & 3324 & 3307 & 3265 \\
\hline 11 & 3287 & 3274 & 3256 & 3170 \\
\hline 12 & 3201 & 3211 & 3182 & 3068 \\
\hline meanD $^{\mathrm{f}, \mathrm{g}}$ & 0 & -0.6 & 1.5 & -1.4 \\
\hline MAD $^{\mathrm{g}}$ & 0 & 9.8 & 26 & 68 \\
\hline maxD $^{\mathrm{g}}$ & 0 & 22 & 45 & 133 \\
\hline
\end{tabular}

${ }^{\mathrm{a}}$ Gas-phase, Table 1 of Diken et al. ${ }^{13}$

${ }^{\mathrm{b}}$ Table V of Kim \& Kim, scaled by $0.938 .{ }^{17}$

${ }^{\mathrm{c}}$ Table $\mathrm{S} 7$ of Temelso et al., harmonic, scaled by $0.9685 .{ }^{19}$

${ }^{\mathrm{d}}$ Table S7 of Temelso et al. (anharmonic). ${ }^{19}$ Unscaled (i.e., scaled by 1.0).

${ }^{\mathrm{e}}$ Bands 1-4 are averaged together.

${ }^{\mathrm{f}}$ Mean deviation (calculated minus experiment).

${ }^{g}$ Calculated with respect to experiment for modes 6,7, 9-12 (i.e., all available HBed-OH stretching modes). Whenever modes 7 and 8 are resolved, they are averaged for making this comparison. 
Table S3. Comparison with experiment of the MB-pol book-isomer OH-stretch spectrum when the shift is determined to generate best agreement with the optimally-scaled quantum chemistry methods of Table $\mathrm{S} 2$.

\begin{tabular}{|l|l|l|l|l|}
\hline mode & Exper. $^{\text {a }}$ & $\begin{array}{l}\text { MB-pol/MP2 } \\
6-311+G^{*} *\end{array}$ & $\begin{array}{l}\text { MB-pol/MP2 } \\
\text { aug-cc-pVDZ }\end{array}$ & $\begin{array}{l}\text { MB- } \\
\text { pol/VPT2 }^{\mathrm{d}}\end{array}$ \\
\hline $1-4^{\mathrm{e}}$ & 3711 & 3707 & 3709 & 3706 \\
\hline 5 & & 3693 & 3695 & 3695 \\
\hline 6 & 3572 & 3584 & 3586 & 3583 \\
\hline 7 & 3464 & 3460 & 3462 & 3459 \\
\hline 8 & & 3452 & 3454 & 3451 \\
\hline 9 & 3423 & 3410 & 3412 & 3409 \\
\hline 10 & 3327 & 3327 & 3329 & 3326 \\
\hline 11 & 3287 & 3278 & 3280 & 3277 \\
\hline 12 & 3201 & 3218 & 3220 & 3217 \\
\hline & & & & \\
\hline MAD $^{\mathrm{f}}$ & 0 & 8.4 & 8.1 & 8.9 \\
\hline maxD $^{\mathrm{f}}$ & 0 & 17 & 19 & 16 \\
\hline
\end{tabular}

${ }^{\mathrm{a}}$ Gas-phase, Table 1 of Diken et al. ${ }^{13}$

${ }^{b}$ MB-pol shifted by $208 \mathrm{~cm}^{-1}$, to obtain best agreement with Table V of Kim \& Kim, ${ }^{17}$ scaled by 0.938 .

${ }^{\mathrm{c}}$ MB-pol shifted by $206 \mathrm{~cm}^{-1}$, to obtain best agreement with Table $\mathrm{S} 7$ of Temelso et al., harmonic frequencies, scaled by $0.9685 .{ }^{19}$

${ }^{\mathrm{d}}$ MB-pol shifted by $209 \mathrm{~cm}^{-1}$, to obtain best agreement with Table S7 of Temelso et al. (anharmonic), ${ }^{19}$ scaled by 1.02 .

${ }^{\mathrm{e}}$ Bands 1-4 are averaged together.

${ }^{\mathrm{f}} \mathrm{MAD}$ and maxD with respect to experiment for modes 6,7, 9-12 (i.e., all available HBed-OH stretching modes). Whenever modes 7 and 8 are resolved, they are averaged for making this comparison. 


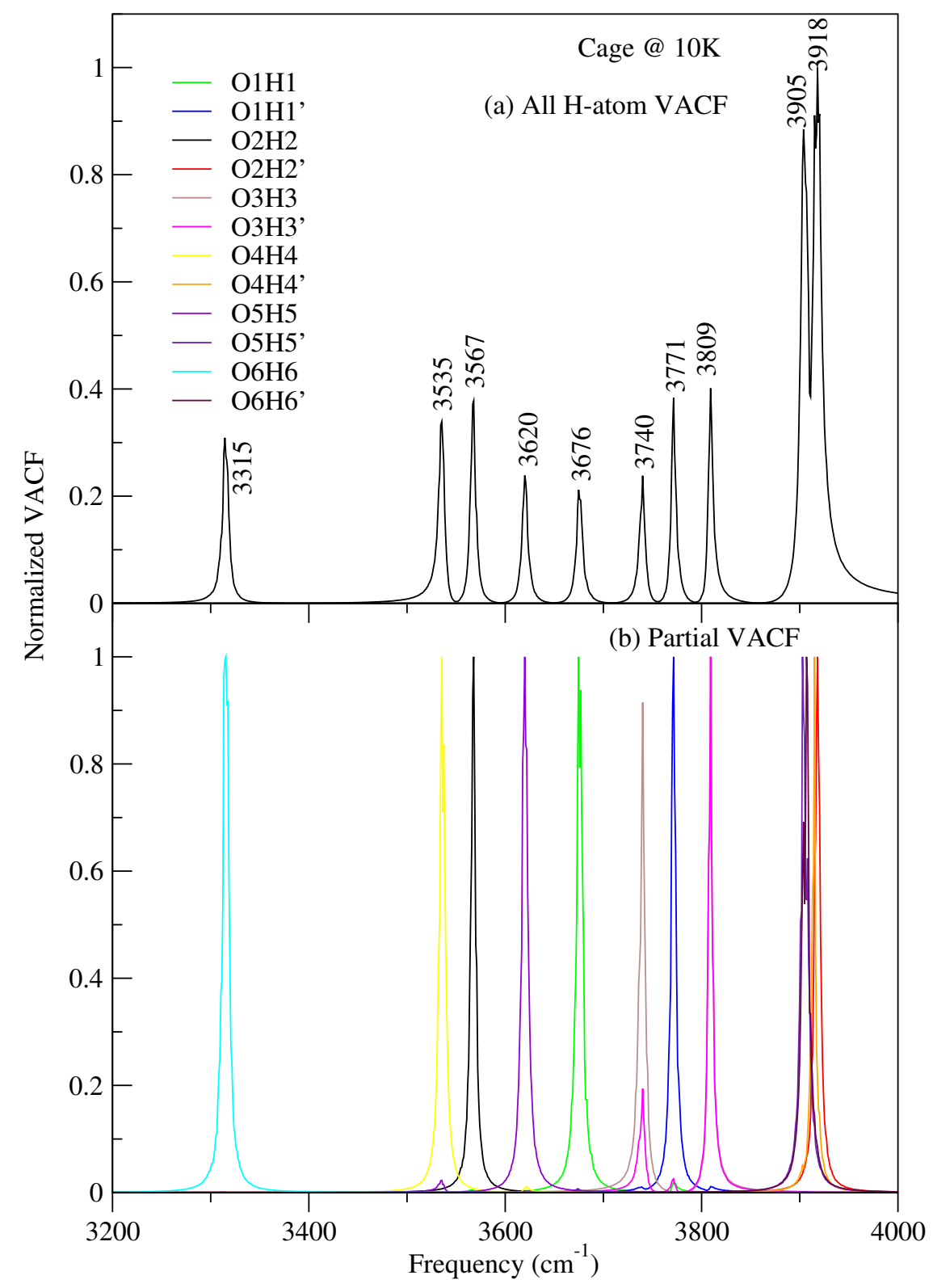

Figure S1: VACF vibrational spectrum of the cage isomer at $10 \mathrm{~K}$ and a $10 \mathrm{~ns}$ length of trajectory, (a) All H-atom VACF and (b) Partial VACF for the $\mathrm{OH}$ distances color coded in panel (a). The LMA of the HBed OH stretches can be read from this figure as follows (low to high) O6H6 (DAA), O4H4 (DAA), O2H2 (DA), O5H5 (DA), O1H1 (DDA), O3H3 (DDA), O1H1' (DDA), O3H3' (DDA). 


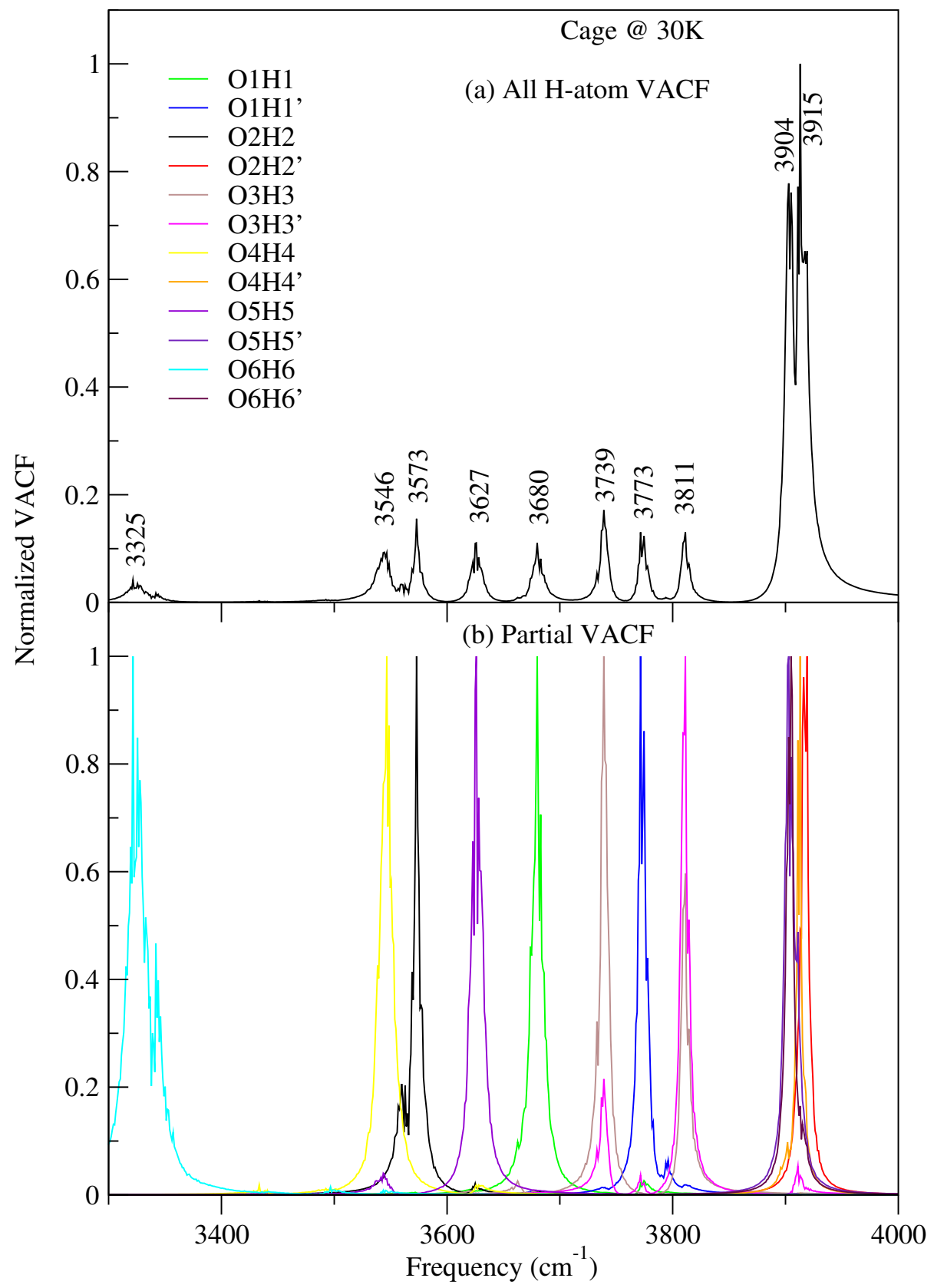

Figure S2: Same as Figure S1 at 30K. 


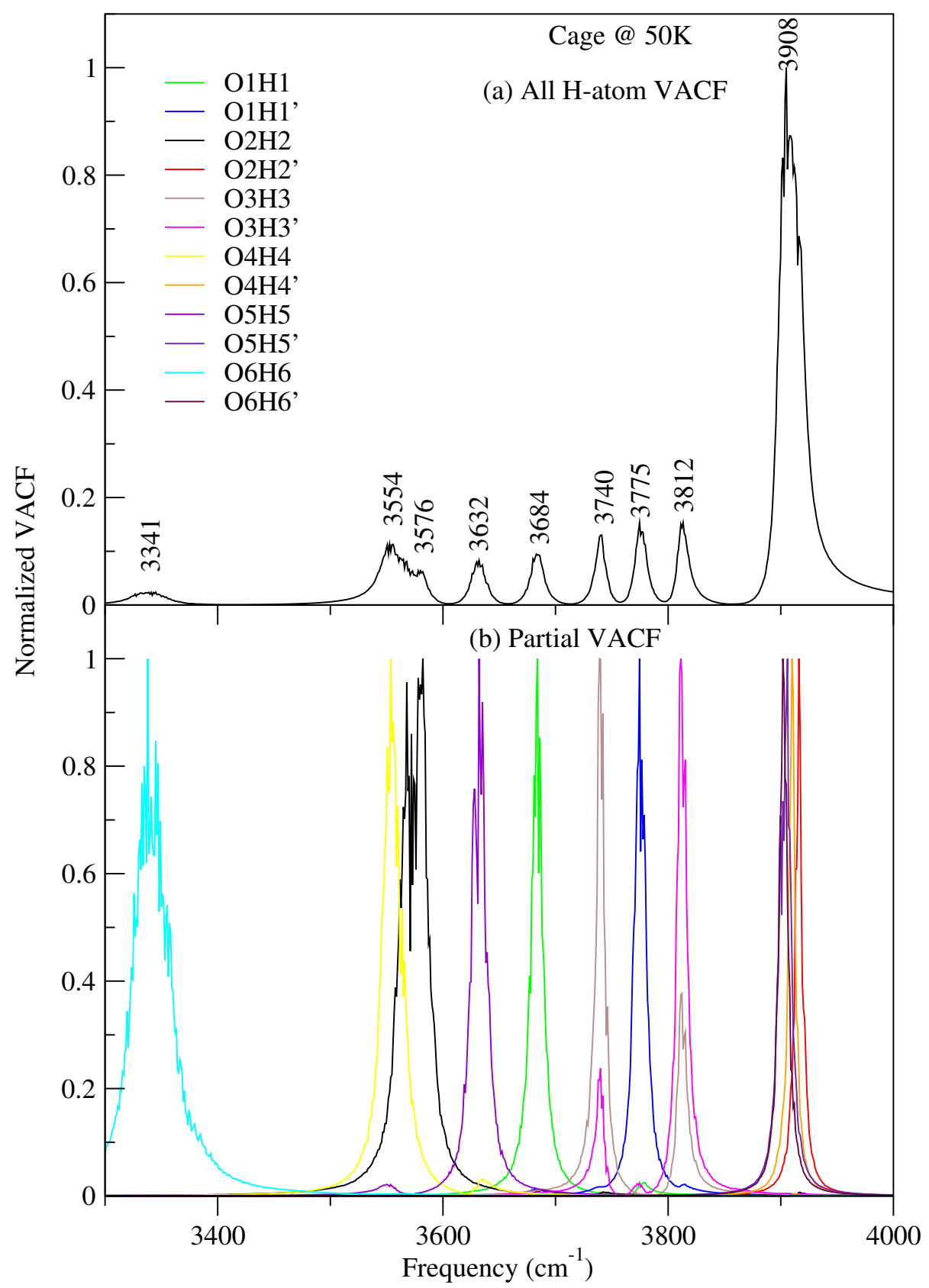

Figure S3: Same as Figure S1 at 50K. 


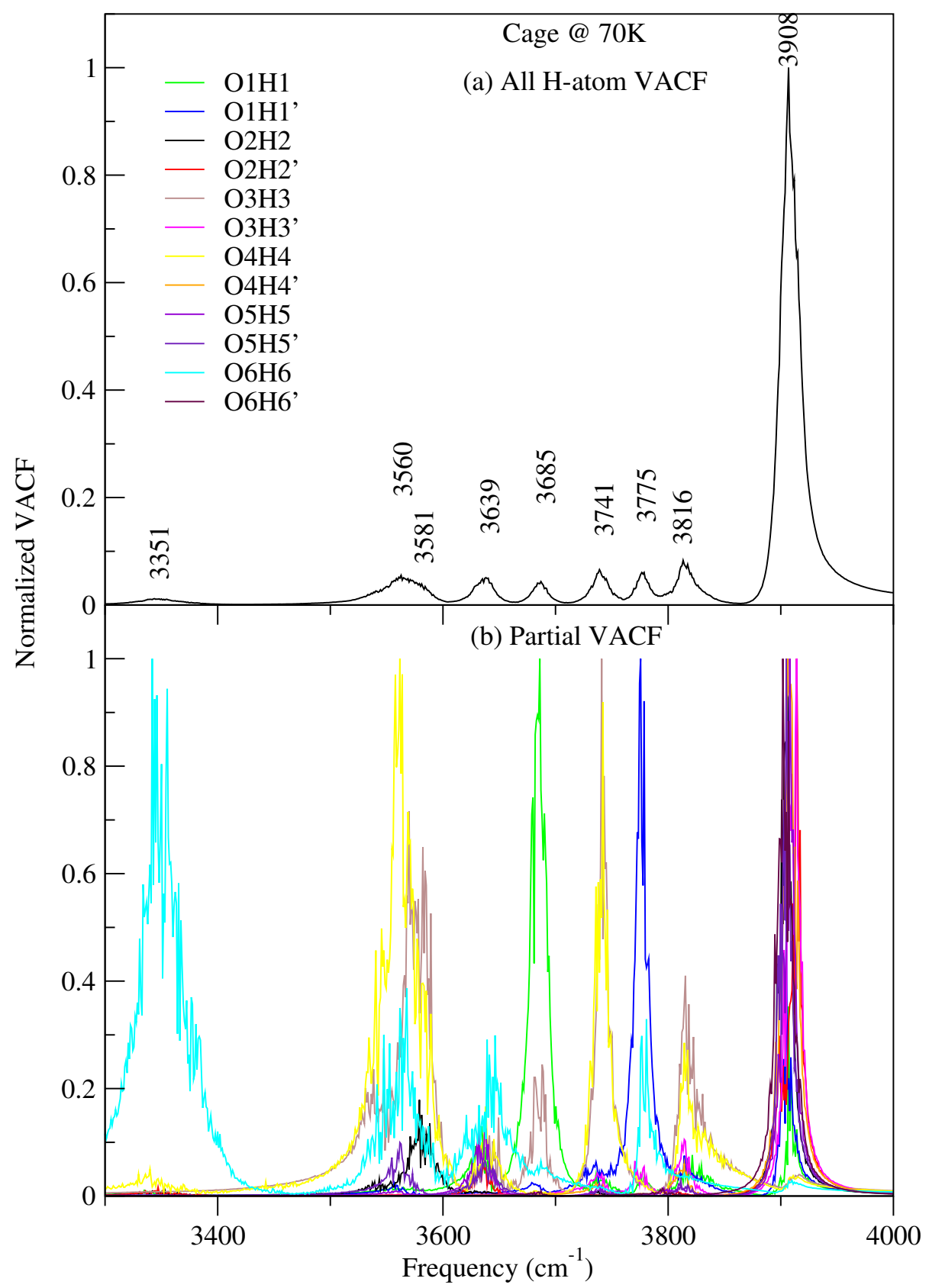

Figure S4: Same as Figure S1 at 70K. 


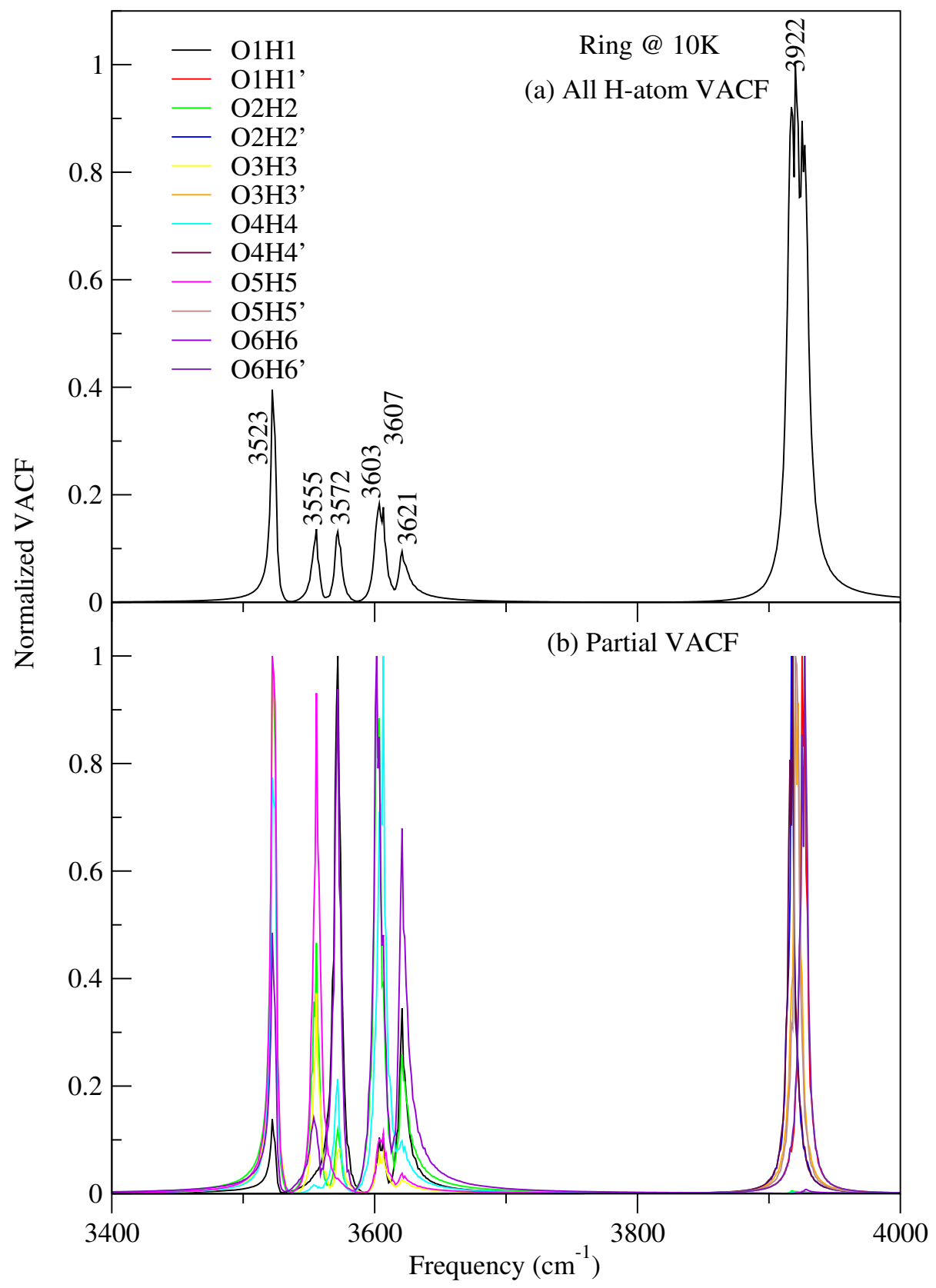

Figure S5: VACF vibrational spectrum of the ring isomer at $10 \mathrm{~K}$ and a $10 \mathrm{~ns}$ length of trajectory, (a) All H-atom VACF and (b) Partial VACF for the $\mathrm{OH}$ distances color coded in panel (a). 


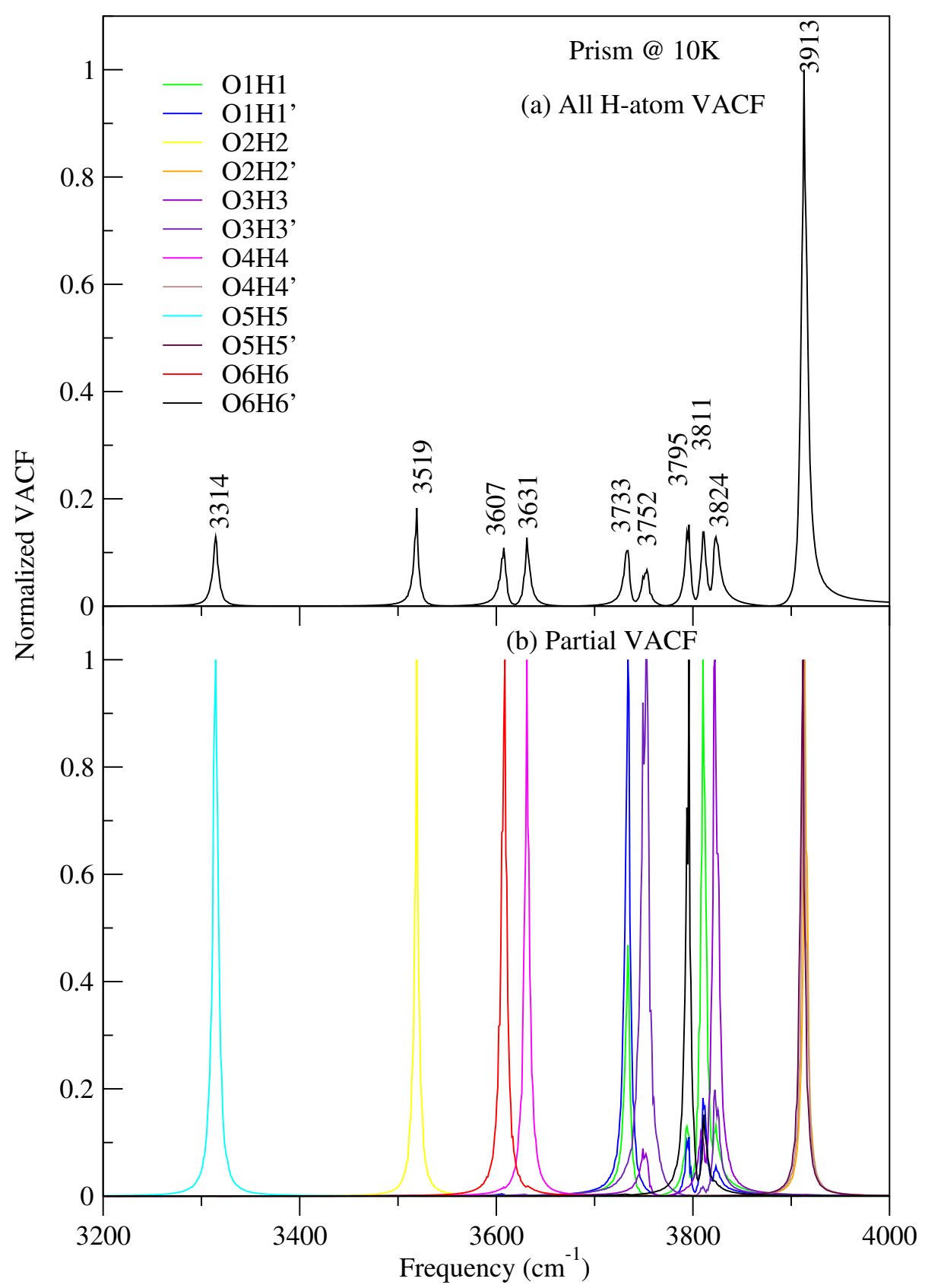

Figure S6: VACF vibrational spectrum of the prism isomer at $10 \mathrm{~K}$ and a $10 \mathrm{~ns}$ length of trajectory, (a) All H-atom VACF and (b) Partial VACF for the $\mathrm{OH}$ distances color coded in panel (a). The LMA of the HBed OH stretches can be read from this figure as follows (low to high) O5H5 (DAA), O2H2 (DAA), O6H6 (DDA), O4H4 (DAA), O1H1' (DDA), O3H3' (DDA), O6H6' (DDA), O1H1 (DDA), O3H3 (DDA). 